\title{
Russell's Viper Venom Clotting Time of Common Carp Plasma
}

\author{
Sung Hee Jung and Hiroshi Kawatsu \\ Fish Pathology Division, Faculty of Agriculture, Miyazaki University, Gakuen-kibanadai, Miyazaki 889-21, Japan
}

(Received December 2, 1993)

The clotting time using Russell's viper venum (RVV) was examined in common carp plasma. The best condition for the assay of RVV time was obtained in a $1: 30,000$ dilution of RVV with Platelin suspension and $\mathrm{M} / 20 \mathrm{CaCl}_{2}$ solution. Common carp plasma was also subjected to chromogenic substrate assay for human factor $\mathrm{X}$. The dilution curves of RVV time (RVVT) and chromogenic substrate assay were made from serial saline dilutions of pooled normal plasma. Reductions in coagulation factors had no marked effects on prolongation of RVV time until reaching a dilution less than approximately $30 \%$. The activity converted from RVVT showed a linear relationship between the activity converted from chromogenic substrate assay within the range from 100 to $40 \%$.

Key words: common carp, blood, blood clotting time

The mechanism of blood coagulation in teleost fish occurs via two pathways, i.e., the intrinsic and the extrinsic pathways, as in mammals. ${ }^{1-3)}$ The intrinsic pathway begins to work by the activation of the contact factor (corresponding to factor XII in humans), and the extrinsic pathway by the extrinsic factor (corresponding to factor VII in humans). These two pathways join to the common pathway, the final step of clot formation, in which prothrombin and fibrinogen are involved. The interaction of these three pathways has been illustrated schematically as a $Y$-shaped figure. Activity of the intrinsic pathway is measured by activated partial thromboplastin time (APTT) and the extrinsic activity by prothrombin time (PT). Strictly speaking, these two clotting times reflect the sum total activities of coagulation factors involved in "intrinsic plus common" or "extrinsic plus common" pathways. To differentiate between the activity of each pathway, it is necessary to separate the activity of the common pathway. In mammals, factor $X$ links the intrinsic and extrinsic pathways and functions as a starting device of the common pathway. Since it was found that Russell's viper venom (RVV) directly activates factor $\mathrm{X}$ without participation of the factors in the intrinsic and extrinsic pathways, RVV clotting time has been used as a parameter to measure common pathway activity. ${ }^{4-7)}$

From the later half of the 1970's, synthetic chromogenic peptide substrates were introduced into coagulation factor assays in mammals. ${ }^{8-10)}$ In this method, RVV is still essential in the activation of factor $X$ to factor $X$ a for the measurement of factor $\mathrm{X}$ activity. Although RVV may be useful for measurement of common pathway activity in fish blood, we found only one report in the literature concerning such as assay, by Lewis ${ }^{11}$ who used elasmobranch blood.

We describe in the present report procedures for determining RVV clotting time (RVVT) and chromogenic substrate assay for common carp plasma and discuss their possible general use for evaluating common pathway activity in fish.

\section{Materials and Methods}

Experiment

Nine parts of blood were drawn from a caudal vessel of the common carp Cyprinus carpio into a plastic syringe containing one part of $3.13 \%$ trisodium citrate solution, the plasma was obtained by centrifugation, and equal volumes of plasma specimens collected from three or four individuals were pooled. Graded dilutions of pooled plasma in saline were prepared and RVVT and chromogenic substrate assay were determined for each dilution. All assays were made in triplicate and their mean values were recorded.

\section{Procedures for Determination of RVVT}

Determination of the Optimal Concentration of $R V V:$ Russell's Viper Venom (Sigma) was dissolved in distilled water to final concentrations ranging from $1: 10,000$ to $1: 320,000$. The $\mathrm{RVV}$ solutions were mixed with an equal volume of $\mathrm{M} / 40 \mathrm{CaCl}_{2}$ solution and preheated in a water bath at $37^{\circ} \mathrm{C}$. Test plasma $(0.1 \mathrm{ml})$ was pipetted into a polystyrene tube $(10 \times 72 \mathrm{~mm})$ and incubated at $37^{\circ} \mathrm{C}$ for $3 \mathrm{~min}$. Then, $0.2 \mathrm{ml}$ of each $\mathrm{RVV}-\mathrm{CaCl}$ mixture was added and the stopwatch was started. The clotting time was taken as the time for fibrin clot formation. A 1:30,000 dilution of RVV gave the shortest clotting time (Table 1).

Determination of the Optimal Concentration of $\mathrm{CaCl}_{2}: \mathrm{RVV}$ was dissolved in distilled water to a concentration of $1: 30,000$, and mixed with an equal volume of $\mathrm{M} / 10, \mathrm{M} / 20$, or $\mathrm{M} / 40 \mathrm{CaCl}_{2}$ solution. The clotting time was determined by the same procedure as described above, and the $\mathrm{M} / 20 \mathrm{CaCl}$. solution gave the shortest clotting time (Table 2).

Furthermore, supplementation of the reagent with Platelin (Organon Teknika Corp., North Carolina, USA) produced a solid fibrin clot and

Table 1. Determination of optimal concentration of Russell's viper venom

\begin{tabular}{ccc}
\hline & \multicolumn{2}{c}{ Clotting time (seconds)* } \\
\cline { 2 - 3 } Concentration of PVV & Plasma 1 & Plasma 2 \\
\hline $1: 10,000$ & 79.7 & 119.5 \\
$1: 20,000$ & 46.6 & 46.0 \\
$1: 30,000$ & 20.1 & 28.6 \\
$1: 40,000$ & 25.1 & 32.7 \\
$1: 80,000$ & 38.1 & 48.6 \\
$1: 160,000$ & 47.9 & 59.9 \\
$1: 320,000$ & 59.1 & 70.7 \\
\hline
\end{tabular}

* Two lots of pooled plasma from 3 fish were examined. 
Table 2. Determination of optimal $\mathrm{CaCl}_{2}$ solution

\begin{tabular}{|c|c|c|c|c|}
\hline \multirow{2}{*}{$\begin{array}{l}\text { Concentration } \\
\text { of } \mathrm{CaCl}_{2}\end{array}$} & \multicolumn{4}{|c|}{ Clotting time (seconds) ${ }^{*}$} \\
\hline & Plasma 1 & Plasma 2 & Plasma 3 & Plasma 4 \\
\hline $1 / 10 \mathrm{M}$ & 56.4 & 64.3 & 60.8 & 35.5 \\
\hline $1 / 20 \mathrm{M}$ & 27.1 & 32.1 & 28.9 & 19.7 \\
\hline $1 / 40 \mathrm{M}$ & 31.3 & 40.3 & 29.2 & 23.9 \\
\hline
\end{tabular}

* Four lots of pooled plasma from 3 fish were examined.

made it easier to determine the clotting time.

Finally, we adopted the following reagent and procedures.

Reagent: RVV was dissolved in distilled water to a concentration of $1: 30,000$, and $2.5 \mathrm{~m} l$ of this solution was added to a vial of Platelin which contained rabbit brain phospholipid $(1.8 \mathrm{mg} / \mathrm{vial})$ and sodium chloride $(13.4 \mathrm{mg} / \mathrm{vial})$. The RVV plus Platelin solution was mixed with an equal volume of $\mathrm{M} / 20 \mathrm{CaCl}_{2}$, solution.

Procedures: Assay was then performed as above.

Procedures for the Chromogenic Substrate Assay

Reagent: The chromogenic substrate method for human factor $\mathrm{X}$ was applied to common carp plasma. A Coatest FX kit was purchased from KabiDiagnostica (Stockholm, Sweden; imported by Dailchi Pure Chemicals Co., Ltd., Tokyo). The substrate solution ( $\$-2337,1.5 \mathrm{mmol} / l$ ) was reconstituted with distilled water. To prepare the $\mathrm{RVV}-\mathrm{CaCl}_{2}$ solution, RVV (Sigma) was dissolved in distilled water to a final concentration of $1: 10,000$, and mixed with an equal volume of $\mathrm{M} / 20 \mathrm{CaCl}_{2}$ solution. The concentrations of $\mathrm{RVV}$ and $\mathrm{CaCl}_{2}$ were modified from those given in the instruction with the kit.

Procedures: The manual end-point technique was used. The substrate solution was preheated in a water bath at $37^{\circ} \mathrm{C}$. Aliquots of $10 \mu l$ of the test plasma were pipetted into two polystyrene tubes $(10 \times 72 \mathrm{~mm}$, one was the specimen and the other was used as a blank) and $200 \mu \mathrm{l}$ of buffer solution was added to each tube. After incubation at $37^{\circ} \mathrm{C}$ for $3 \mathrm{~min}, 200 \mu l$ of substrate solution was added to the specimen tube, then $200 \mu \mathrm{l}$ of $\mathrm{RVV}-\mathrm{M} / 20 \mathrm{CaCl}_{2}$ solution was added within $30 \mathrm{~s}$. After exactly $3 \mathrm{~min}$, $200 \mu l$ of $50 \%$ acetic acid was added to both tubes. Finally, $400 \mu l$ of distilled water was added to the blank tube, and the absorbance at $405 \mathrm{~nm}$ was measured against the blank.

\section{Results}

\section{RVVT of Common Carp Plasma}

The RVVT of common carp plasma obtained from 89 healthy fish ranged from $16.6 \mathrm{~s}$ to $34.0 \mathrm{~s}$, and the mean \pm S.D. was $24.8 \pm 4.0 \mathrm{~s}$.

\section{RVVT and Chromogenic Substrate Assay of Graded Dilutions of Plasma}

Measurements of RVVT and chromogenic substrate assay were made from serial dilutions of pooled plasma in saline (Figs. 1 and 2). Dilution curves of RVVT and chromogenic substrate assay were expressed by the following equations:

$$
\begin{array}{ll}
Y=158.6452 X^{-0.46323} & (r=0.99) \\
Y=0.01564 X^{0.36680} & (r=0.99)
\end{array}
$$

where $Y$ indicates the clotting time or absorbance, and $X$ represents the percentage of dilution. The RVVT of graded dilutions of plasma was markedly prolonged with dilutions of $20 \%$ or less (Fig. 1). In the chromogenic substrate assay, absorbances of graded dilutions of plasma gradually decreased with increases in dilution (Fig. 2). When measured using distilled water instead of plasma, the absorbance in the chromogenic substrate assay was $0.026 \pm 0.002$.

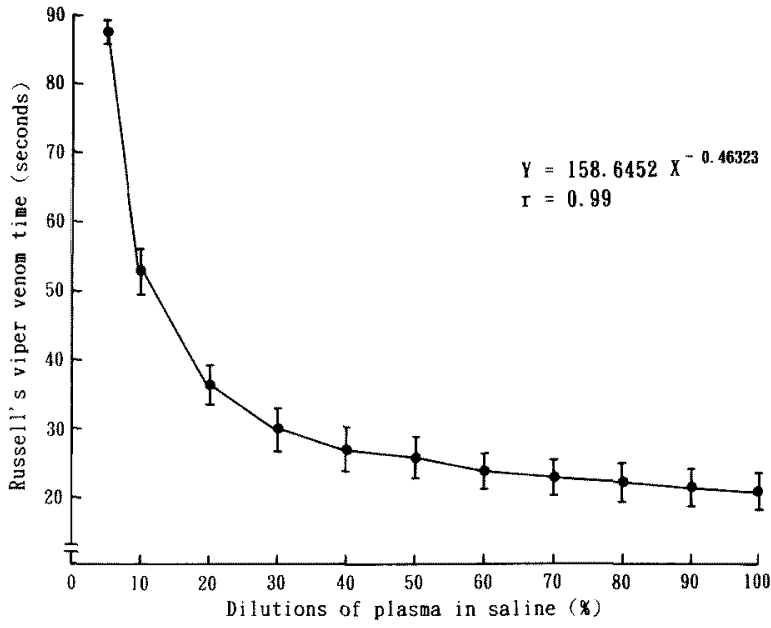

Fig. 1. Dilution curve of RVVT in serial saline dilutions of normal pooled plasma.

The clotting time at higher dilutions of pooled plasma of $20 \%$ or less was determined as the time when the first fibrin web appeared. Each symbol with a vertical bar represents the mean \pm S.D. obtained from five lots of pooled plasma.

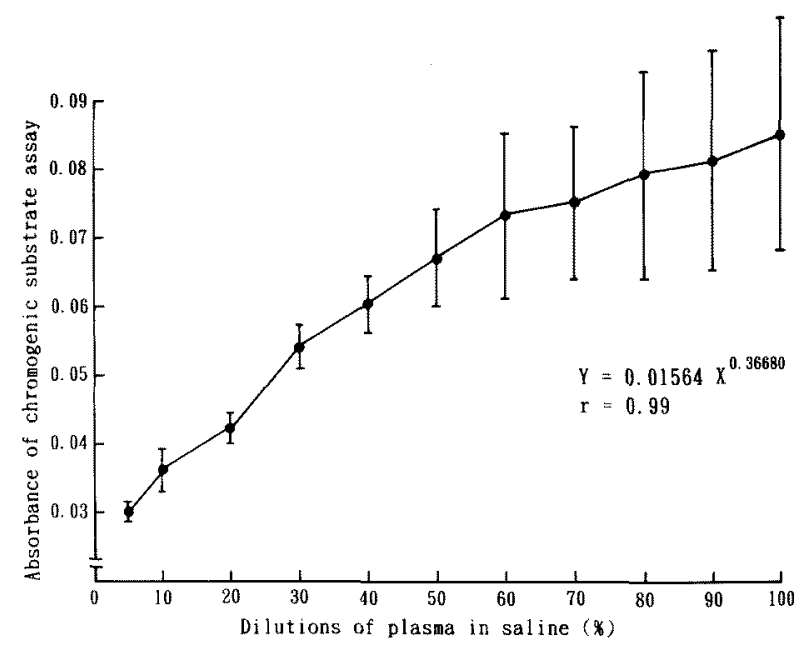

Fig. 2. Dilution curve of chromogenic substrate assay in serial saline dilutions of normal pooled plasma.

Each symbol with a vertical bar represents the mean \pm S.D. obtained from five lots of pooled plasma.

\section{Relationship between RVV Activity and Chromogenic Activity}

According to the conventional method used in clinical hematology, each clotting time or absorbance of a test plasma was converted to percentage activity from each dilution curve. In each curve, the clotting time or absorbance of the original pooled plasma represents $100 \%$ activity. Thus, the activity could be calculated by the following equations:

$$
\text { RVV activity }=(\text { RVVT } \div 158.6452)^{-2.15875}
$$

Chromogenic activity $=(\text { absorbance } \div 0.01564)^{2.72628}$

Figure 3 shows the correlation diagrams between RVV activity and chromogenic activity. A linear relationship was 


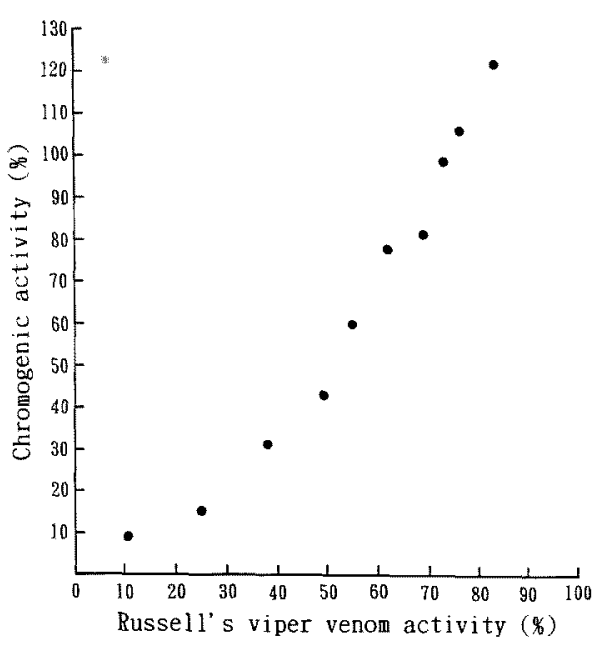

Fig. 3. Correlation between RVV activity (\%) and chromogenic activity $(\%)$ in serial saline dilutions of normal pooled plasma.

seen within the range from $100 \%$ to approximately $40 \%$.

\section{Discussion}

The shortest clotting time was obtained in a $1: 30,000$ dilution of $\mathrm{RVV}$ and $\mathrm{M} / 20 \mathrm{CaCl}_{2}$ solution. The optimal conditions for RVVT measurement for common carp plasma were approximately equivalent to those for human plasma. ${ }^{4-6)}$ The end point of clot formation became sharp when Platelin was added to the RVV solution, and this also agreed with findings obtained using human plasma. ${ }^{4-7)}$

However, RVVT of common carp plasma $(24.8 \pm 4.0 \mathrm{~s})$ was relatively long compared with $4.8 \pm 0.3 \mathrm{~s}$ in $\mathrm{PT}^{(2)}$ and $4.9 \pm 0.5 \mathrm{~s}$ in APTT. ${ }^{3)}$ When compared with clotting times in human plasma ${ }^{13)}(11-15 \mathrm{~s}$ in PT, 28-38 $\mathrm{s}$ in APTT, and $12-16 s$ in RVVT), RVVT of common carp was considerably longer. This suggests that the activity of RVV is not as strong on teleost plasma. Although Lewis ${ }^{11)}$ reported that RVV had no effect on recalcification time in the skate Raja eglanteria, the present results indicate that $\mathrm{RVV}$ is a potent accelerator of recalcification time in teleost plasma. The pattern of the dilution curve of RVVT in common carp plasma was also similar to that of human plasma, in which the decrease of factor $X$ has no effect on prolongation of clotting time until about $30 \%$ of normal. ${ }^{5)}$ These similarities of RVVT observed between common carp and human plasma support the existence of an X-like factor in common carp plasma.

The chromogenic substrate assay has several advantages over the clotting method because it is possible to select the substrate which has the greatest affinity for a specified coagulation factor and to avoid interference due to the effects of other factors. In addition, this method requires very a small volume plasma sample $(20 \mu l$ in total). Although the existence of factor $\mathrm{X}$ has not been demonstrated in fish, the linear relationship between activities of RVV and chromogenic substrate assay suggests that chromogenic substrate S-2337 is also available for the measurement of factor X-like activity in fish.

Recently, Smit and Schoonbee ${ }^{14)}$ employed human factor-deficient plasmas to demonstrate fish coagulation factors, and emphasized that the clotting factors are similar to those of mammalian or human blood. However, the results of their work seem to contradict the results of previous works, ${ }^{1,2)}$ in which it was pointed out that congenitally deficient mammalian plasma substrates can not be used to assay clotting factors in non-mammalian species wihtout specific proof of validity. We have also obtained negative results when we tried to use human factor VII deficient plasma in the common carp plasma (unpublished). We therefore consider it appropriate to use trusted procedures in research on the fish-blood coagulation system, even if these procedures are now out-of-date in research on human blood.

\section{References}

1) R. F. Doolittle and D. M. Surgenor: Blood coagulation in fish. Am. J. Physi., 203, 964970 (1962)

2) C. Hougie: Coagulation changes in healthy and sick Pacific salmon. Adv. Exp. Med. Biol., 22, 89-102 (1971).

3) H. Kawatsu and M. Sato: Effect of glass contact on the clotting time of carp blood. Nippon Suisan Gakkaishi, 53, 1533-1536 (1987).

4) P. Hjort, S. I. Rapaport, and P. A. Owren: A simple, specific one-stage prothrombin assay using Russell's viper venom in cephalin suspension. J. Lab. \& Clin. Med., 46, 89-97 (1955).

5) C. Hougie: Effect of Russell's viper venom (Stypven) on Stuart clotting defect. Proc. Soc. Exp. Biol., 93, 570-573 (1956).

6) F. Bachmann, F. Duckert, and F. Koller: The Stuart-Prower factor assay and its clinical significance. Thromb. Diath. Haemorrh., 2, 24-38 (1958)

7) K. W. Denson: The specific assay of Prower-Stuart factor and factor VII. Acta Haemat, 25, 105-120 (1961).

8) L. Aurell, P. Friberger, G. Karlsson, and G. Claeson: A new sensitive and highly specific chromogenic peptide substrate for factor $\mathrm{Xa}$. Thromb. Res., 11, 595-609 (1977).

9) K. Bergström and N. Egberg: Determination of vitamin K sensitive coagulation factors in plasma. Studies on three methods using synthetic chromogenic substrates. Thromb. Res., 12, 531-547(1978).

10) E. M. van Wijk, L. H. Kahlé, and J. W. ten Cate: A rapid manual chromogenic factor X assay. Thromb. Res., 22, 681-686 (1981).

11) J. H. Lewis: Comparative hemostasis. Studies on elasmobranchs. Comp. Biochem. Physiol., 42A, 233-240 (1972).

12) H. Kawatsu and K. Kondo: Prothrombin time of common carp blood. Nippon Suisan Gakkaishi, 55, 183 (1989).

13) M. Matsuoka: Hemorrhagic diathesis and thrombosis, 2nd ed., Kanehara Co., Ltd., Tokyo, 1981, pp. $86-89$ (in Japanese).

14) G. L. Smit and H. J. Schoonbee: Blood coagulation factors in the freshwater fish Oreochromis mossambicus. J. Fish. Biol., 32, 673-677 (1988) 